\title{
Patient facing technologies to improve patient/family engagement in inpatient and outpatient settings
}

\author{
Onur Asan \\ Department of Medicine \\ Medical College of Wisconsin \\ Milwaukee, WI \\ oasan@mcw.edu
}

\begin{abstract}
This medical perspective abstract is about two ongoing projects regarding patient centered information technology designs in inpatient and outpatient settings. These novel technologies aim to improve patient/family engagement by empowering them via information technology. We also reported some of the initial results.
\end{abstract}

Keywords - Patient Centred HIT, Family engagement

\section{BACKGROUND}

Health care has been shifting from a paternalistic model to a patient/family centered model with an appropriate focus on patient/family engagement and empowerment. Recently, there has been increased attention on the potential role of Health Information Technologies (HIT) in patient/family engagement outcomes. In theory, HIT can facilitate patient access to personal records, test results, and health education tools, which can in turn help improve patient/family engagement and empowerment. Recently, there is an increased focus on patient engagement and patient facing technologies, which enable and facilitate patient access to data in health care settings. Some studies tried to present new patient engagement technology applications in adult emergency departments, such as a mobile phone application that provides dynamic information to the patient, a tablet computer which enable patients to access EHR system, and finally a study assessed usefulness of a paper prototype of in-room information display in an emergency department. In addition, several studies also explored how screen sharing with patients influenced patient empowerment and doctor-patient communication in outpatient settings. The purpose of this short proposal is to report two ongoing projects which have being used patient facing technologies to improve patient understanding of care and patient/family engagement in patient setting (Pediatrics Intensive Care Unit) and outpatient settings (primary care exam room).

\section{OBJECTIVE}

The first project was designing a patient centric HIT which can improve patient-doctor communication, patient understanding of the data and patient engagement in the primary care visit. Our previous work from the analysis of 150 videotape visits with using lag sequential analysis showed that computers have impact on doctor-patient communication cues. Different information sharing styles using HIT were identified. The new set up includes a separate screen for patients which will be controlled by physicians and show patient data such as medical history, data, trends, tables with minimizing unnecessary clutter on a traditional providers HIT screen. Physicians used the second screen to educate patients with using risk calculators. We explored how this new set up influence patient education, understanding of care and patient engagement.

The second project was about a novel technology implemented in ICU. This is a 42 " flat panel touch screen monitor that displays validated patient information including vital signs, laboratory results, medications, and interventions in each patient room in Pediatrics ICU. In contrast to traditional HIT intended for providers that requires a secure log-in in order to access patient information, this monitor is available in each patient's room for viewing and use by providers as well as patients/families without any login required. Instead, this monitor is activated upon admission of a patient. Additionally, the content of the monitor is customizable by the organization. The goal of this study was to understand perception and use of this novel technology, as well as identify unmet needs of providers and families to improve the current design of this novel technology.

\section{RESULTS AND DISCUSSION}

The initial analysis revealed several themes. The identified themes were: physicians' familiarity with the monitor, their use routines (when they use it or not), perceived positive/negative properties of the monitor including perceived usefulness and ease of use, its contribution to rounds, how it influenced their interactions with patients' families, their perception of how families use or the monitor or not on their own, implications for family engagement, and suggestions for design improvements.

Patient/families and providers in intensive care unit must contend with complex health information in a demanding health care setting. Patient facing applications have great potential to support patient/family-centered care by providing personalized, accurate, accessible, and actionable health information. Indeed, the findings of this exploratory study can guide future redesign of patient centered HIT and intervention studies while also providing a framework for investigating other transformative HIT applications. 